\title{
A Rare Case of Lethal Prenatal-Onset Infantile Cortical Hyperostosis
}

\author{
Susan Taejung Kim, Hyeseon Kim, Hyun Ho Kim, Na Hyun Lee, Yeaseul Han, \\ Se In Sung, Yun Sil Chang, and Won Soon Park \\ Department of Pediatrics, Samsung Medical Center, Sungkyunkwan University School of Medicine, Seoul, Korea.
}

\begin{abstract}
Infantile cortical hyperostosis, or Caffey's disease, usually presents with typical radiological features of soft tissue swelling and cortical thickening of the underlying bone. The disease can be fatal when it presents antenatally, especially before a gestational age of 35 weeks. This fatal, premature form of the disease is known to occur in various ethnic groups around the globe, and approximately 30 cases have been reported in English literature. This paper is unique in that it is the first paper to report a lethal form of prenatal-type infantile cortical hyperostosis diagnosed in South Korea. Born at gestational age of 27 weeks and 4 days, the patient had typical features of polyhydramnios, anasarca, hyperostosis of multiple bones, micrognathia, pulmonary hypoplasia, and hepatomegaly. The patient was hypotonic, and due to pulmonary hypoplasia and persistent pulmonary hypertension, had to be supported with high frequency ventilation throughout the entire hospital course. Due to the disease entity itself, as well as prolonged parenteral nutrition, liver failure progressed, and the patient expired on day 38 when uncontrolled septic shock was superimposed. The chromosome karyotype of the patient was normal, 46, XX, and COL1A1 gene mutation was not detected.
\end{abstract}

Key Words: Infantile cortical hyperostosis, preterm infant, case reports

\section{INTRODUCTION}

Infantile cortical hyperostosis is an inflammatory process that leads to cortical thickening of the bones and swelling of the surrounding soft tissues. ${ }^{1,2}$ There are two known categories of the disease: infantile form, which presents before 5 months of age, and the prenatal type. ${ }^{1-3}$ The infantile form self-subsides within one or two years of supportive care, ${ }^{4}$ and mutation of the $C O L 1 A 1$ gene that codes for alpha-1 type I collagen has been discovered in certain familial cases.,

The prenatal form can be divided into two subgroups. The mild form, with an onset age of after 35 weeks of gestational

\footnotetext{
Received: February 20, 2019 Revised: March 13, 2019

Accepted: March 14, 2019

Corresponding author: Yun Sil Chang, MD, PhD, Department of Pediatrics, Samsung Medical Center, Sungkyunkwan University School of Medicine, 81 Irwon-ro, Gangnam-gu, Seoul 06351, Korea.

Tel: 82-2-3410-3528, Fax: 82-2-3410-0043, E-mail: yschang@skku.edu

-The authors have no potential conflicts of interest to disclose.

(C) Copyright: Yonsei University College of Medicine 2019

This is an Open Access article distributed under the terms of the Creative Commons Attribution Non-Commercial License (https://creativecommons.org/licenses/ by-nc/4.0) which permits unrestricted non-commercial use, distribution, and reproduction in any medium, provided the original work is properly cited.
}

age, usually does not have any associated complications. The severe form, however, has an onset age of before 35 weeks of gestational age, and the patients usually present with polyhydramnios antenatally and anasarca, hydrops, and pulmonary hypoplasia. ${ }^{1,3}$ Less than 500 cases of the prenatal type have been reported, and among them less than 50 cases were of the lethal form. ${ }^{7}$ We report the first known case of the lethal form of prenatal-type infantile cortical hyperostosis diagnosed in South Korea.

\section{CASE REPORT}

The patient was the second child of a previously healthy, nonconsanguineous couple. The couple had a healthy, 6-year-old son. The mother, who was 37 years old and pregnant for 27 weeks and 3 days, was transferred to our institute due to uncontrolled labor, polyhydramnios, and suspected fetal hydrops on antenatal sonography. The infant was born at a gestational age of 27 weeks and 4 days. The infant was edematous, weighing 1970 g, nearly 1.8 times the ideal body weight. Initial height and head circumference was $37 \mathrm{~cm}$ (50 percentile) and $30 \mathrm{~cm}$ (>90 percentile), respectively, which were likely overestimated 
due to skin edema. Aeration was decreased in both lung fields, and the infant had angulated lower arms and an abnormal face with micrognathia and retrognathia. Complete blood cell count and chemistry of the blood sampled from the cord were unremarkable other than mild aspartate aminotransferase and C-reactive protein (CRP) elevation (Table 1).

Initial chest radiograph revealed small lung volume with increased parenchymal opacity. A chest tube was inserted in the left pleural space, expecting drainage of pleural effusion, but nothing was drained, and the tube was removed on day 3 . The initial echocardiogram revealed neither pericardial effusion nor major cardiac anomalies. Due to persistent pulmonary hypertension, high frequency ventilation, inhalation of nitric oxide, and continuous infusion of inotropics was introduced. Mild hepatomegaly with dilated hepatic and portal veins was seen on the abdominal ultrasonography performed on day 1 . Hepatomegaly progressed, and liver function deteriorated because of prolonged parenteral nutrition and the natural course of the disease. Progression of hepatomegaly led to further decrease in lung volume and, thus, overall respiratory function.

The infant displayed no self-movement other than occasional eye blinking and eyeball movement. Ultrasonographic evaluation of the brain revealed mild atrophy and immature gyral pattern of the brain. Electroencephalography performed on day 18 and 25 was normal.

Typical of infantile cortical hyperostosis, skeletal survey showed periosteal reaction with new bone formation and cortical thickening involving the humerus, radius, ulna, femur, tibia, scapula, orbital rim, mandible, and skull base. Chest volume was small and bell shaped with excessive fanning of the lower ribs. No pathological fracture was seen (Figs. 1 and 2).

Table 1. Laboratory Findings on the Day of Birth and Hospital Day 29 When Sepsis was Suspected

\begin{tabular}{|c|c|c|}
\hline & HD 1 (cord blood) & HD 29 \\
\hline \multicolumn{3}{|l|}{ Complete blood cell count } \\
\hline WBC $(/ \mu \mathrm{L})$ & 16370 & 27250 \\
\hline Neutrophil (\%) & 47 & 51 \\
\hline Lymphocytes (\%) & 32 & 16 \\
\hline Hemoglobin (g/dL) & 12.5 & 7.7 \\
\hline 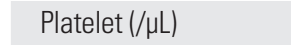 & 207000 & 64000 \\
\hline \multicolumn{3}{|l|}{ Chemistry } \\
\hline Protein/Albumin (g/dL) & $3.9 / 2.4$ & $2.5 / 1.3$ \\
\hline Total Bilirubin (mg/dL) & 1.7 & 12.2 \\
\hline AST/ALT/ALP (U/L) & 101/10/92 & $78 / 95 / 73$ \\
\hline $\mathrm{BUN} / \mathrm{Cr}$ (mg/dL) & $13.8 / 1.03$ & $114.9 / 1.23$ \\
\hline $\mathrm{Ca} / \mathrm{P}(\mathrm{mg} / \mathrm{dL})$ & $8.9 / 5.5$ & $10.5 / 5.4$ \\
\hline $\mathrm{CRP}$ (mg/dL) & 1.11 & 5.65 \\
\hline PT (INR) & - & 1.61 \\
\hline
\end{tabular}

HD, hospital day; WBC, white blood cell; AST, aspartate aminotransferase; ALT, alanine aminotransferase; ALP, alkaline phosphatase; BUN, blood urea nitrogen; $\mathrm{Cr}$, creatinine; CRP, C-reactive protein; PT, prothrombin time; INR, international normalized ratio.
Chromosome karyotype was normal, 46, XX, and COL1A1 gene mutation was not detected.

The initial infection surveillance was unremarkable. However, on day 29, follow-up laboratory results indicated increases

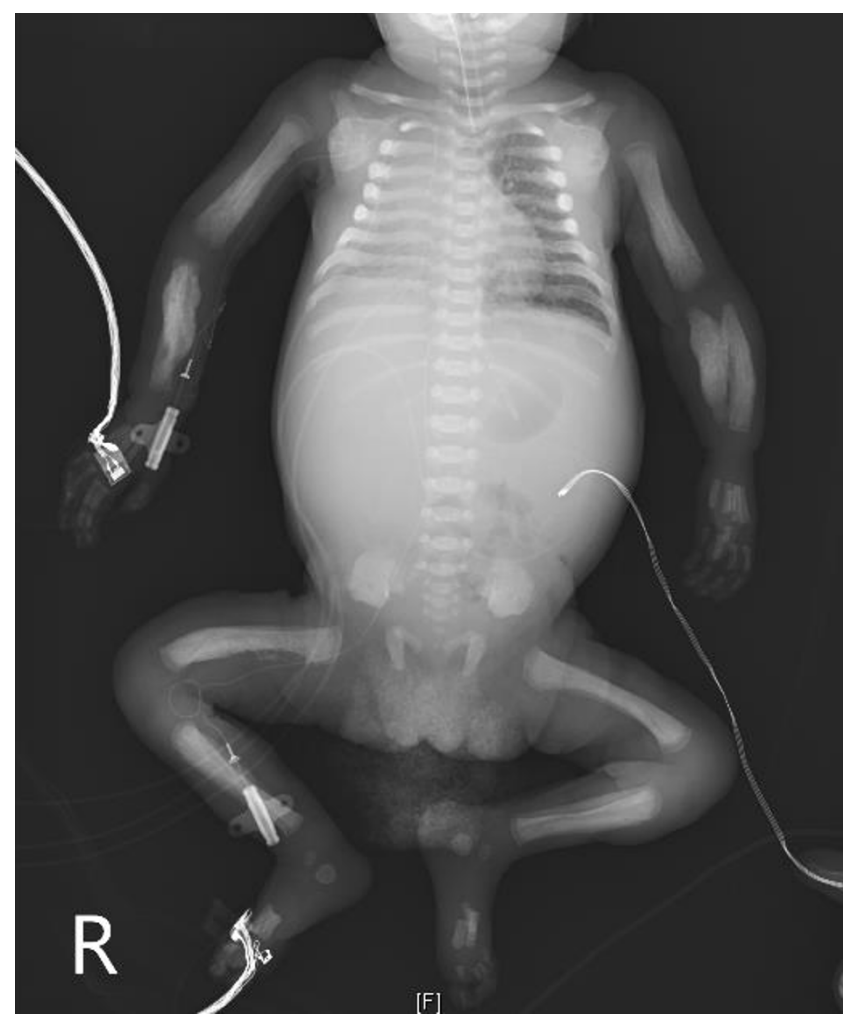

Fig. 1. Infantogram showing cortical hyperostosis of the bilateral humerus, radius, ulna, femur, tibia, and scapula. Note that the chest volume is relatively small and bell shaped with excessive fanning of the lower ribs.

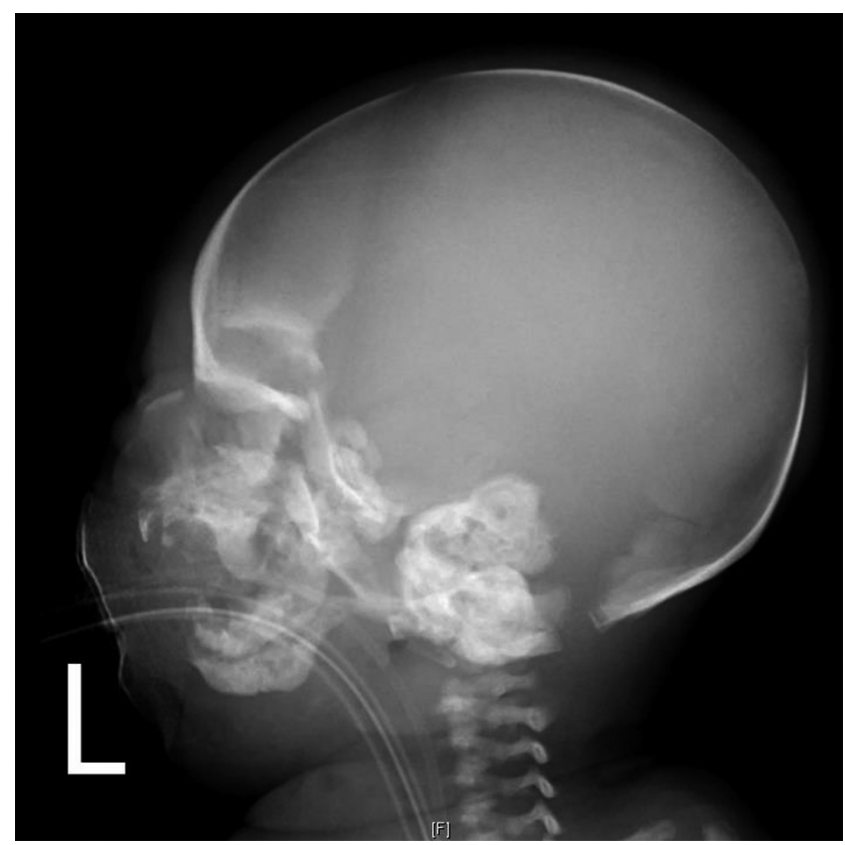

Fig. 2. Skull radiography showing cortical hyperostosis of the bilateral orbital rim, mandible, and skull base. 
in CRP and white blood cell count (Table 1). Blood pressure dropped and broad-spectrum antibiotics were added to treat septic shock. Enterococcus faecalis was isolated from the bloodstream culture.

Due to the disease entity itself, as well as prolonged parenteral nutrition, liver failure progressed, and the patient expired on day 38 when uncontrolled sepsis was superimposed.

This study was approved for exemption of subject consent by Samsung Medical Center Institutional Review Board (Assignment number SMC 2019-01-120-001).

\section{DISCUSSION}

PubMed database search using the keywords Caffey, infantile cortical hyperostosis, lethal, prenatal, and antenatal resulted in 28 cases of the lethal form of prenatal-onset infantile cortical hyperostosis published in English. We report the first known case thereof in South Korea. The patient, born at a gestational age of 27 weeks and 4 days, was the second youngest to survive for over a month among the 28 patients. The patient displayed typical features of polyhydramnios, anasarca, hyperostosis of multiple bones, micrognathia, pulmonary hypoplasia, and hepatomegaly.

According to Kamoun-Goldrat, et al., ${ }^{1} 40 \%$ of the infants affected by this disease are stillborn, $25 \%$ have pulmonary hypoplasia, and about half of livebirths die due to respiratory problems. In our case, the patient died of septic shock due to E. faecalis bacteremia in combination with persistent respiratory problem and progressing hepatic failure. In another case by Al-Harbi, et al., ${ }^{4}$ the patient was hypoactive and hypotonic with known lissencephaly. Our case was similar in that the patient was hypotonic with no recognizable movement other than eye blinking and that she had an immature gyral pattern on brain sonography. Neurologic symptoms related to the disease are not well studied and require further investigation.

Hypoxic damage of the periosteum results from inherited defects of the arterioles, leading to intense proliferation of subperiosteal cells and fibrosis of the bone marrow, which subsequently results in hepatic extramedullary hematopoiesis and portal hypertension. Hepatomegaly and thickening of the ribs limit space for lung development causing pulmonary hypoplasia. ${ }^{3,4}$ The better prognosis when the onset time is beyond gestational age of 35 weeks is because at later ages myeloid hyperplasia more likely occurs in the bone marrow. ${ }^{3}$ Unfortunately, we were not able to perform autopsy on the patient and were not able to confirm the proposed mechanism pathologically.

In conclusion, the prenatal form of infantile cortical hyper- ostosis that presents before the gestational age of 35 weeks can be fatal due to prematurity, as well as the natural course of the disease. The number of cases is still limited, and the pathophysiology of the disease is still under investigation. Identifying the etiology of the disease could help prevent mortality in the future.

\section{ACKNOWLEDGEMENTS}

Supported by Samsung Medical Center (20 by 20 Project, Best \#3, GFO1150091).

\section{AUTHOR CONTRIBUTIONS}

Investigation: Kim ST, Kim H, Kim HH, Lee NH, Han Y, Sung SI, Chang YS, Park WS. Writing-original draft: Kim ST. Writing-reviewing \& editing: Kim ST, Sung SI, Chang YS, Park WS.

\section{ORCID iDs}

Susan Taejung Kim https://orcid.org/0000-0002-0096-7364 Hyeseon Kim https://orcid.org/0000-0002-3909-2737 Hyun Ho Kim https://orcid.org/0000-0002-7379-6041 Na Hyun Lee https://orcid.org/0000-0002-6063-3067 Yeaseul Han https://orcid.org/0000-0002-4074-2936 Se In Sung https://orcid.org/0000-0002-8717-6142 Yun Sil Chang https://orcid.org/0000-0001-9201-2938 Won Soon Park https://orcid.org/0000-0002-8245-4692

\section{REFERENCES}

1. Kamoun-Goldrat A, Martinovic J, Saada J, Sonigo-Cohen P, Razavi F, Munnich A, et al. Prenatal cortical hyperostosis with COL1A1 gene mutation. Am J Med Genet A 2008;146A:1820-4.

2. Oh BL, Paik SW, Kim WS. A case of sporadic Caffey's disease (infantile cortical hyperostosis) without mandibular involvement. Korean J Pediatr 2000;43:1294-9.

3. Wright JR Jr, Van den Hof MC, Macken MB. Prenatal infantile cortical hyperostosis (Caffey's disease): a 'hepatic myeloid hyperplasiapulmonary hypoplasia sequence' can explain the lethality of early onset cases. Prenat Diagn 2005;25:939-44.

4. Al-Harbi M, Barakat N, Shabaan W, Yousef H. Prenatal Caffey disease case report and review of the literature. Med J Cairo Univ 2014;82:625-8.

5. Cerruti-Mainardi P, Venturi G, Spunton M, Favaron E, Zignani M, Provera S, et al. Infantile cortical hyperostosis and COL1A1 mutation in four generations. Eur J Pediatr 2011;170:1385-90.

6. Cho TJ, Moon HJ, Cho DY, Park MS, Lee DY, Yoo WJ, et al. The c.3040C > T mutation in COL1A1 is recurrent in Korean patients with infantile cortical hyperostosis (Caffey disease). J Hum Genet 2008;53:947-9.

7. Saggar K, Shivran N, Saggar K, Ahluwalia A. Infantile cortical hyperostosis or Caffey's disease. A case report and review of the literature. Int J Recent Sci Res 2017;6:213. 